INPLASY

PROTOCOL

To cite: Huang et al. Early nonresponse to neoadjuvant chemotherapy will increase the recurrence of cervical cancer: a system review and metaanalysis. Inplasy protocol 202220107. doi: 10.37766/inplasy2022.2.0107

Received: 23 February 2022

Published: 24 February 2022

Corresponding author: Kecheng Huang

huangkc@hust.edu.cn

Author Affiliation: HUST

Support: nsfc.

Review Stage at time of this submission: Data analysis.

Conflicts of interest:

None declared.

\section{Early non-response to neoadjuvant chemotherapy will increase the recurrence of cervical cancer: a system review and meta-analysis}

Huang, $\mathrm{K}^{1} ; \mathrm{Wu}, \mathrm{X}^{2}$; Chen, $\mathrm{Z}^{3}$.

Review question / Objective: Background: Neoadjuvant chemotherapy has been used for treatment of cervical cancer for a long time; however, the role of early non-response on prognosis is still confusing. This study was designed to assess its impact on disease-free survival (DFS). Methods: Databases "PubMed", "Embase" and the "Cochrane Library" were searched out through May 2020, and both random effects model and fixed effect model were employed to calculate the main pooled results. 12 and Cochrane $Q$ test were used to test the heterogeneity among the studies. Funnel plot with Begg's and Egger's tests was used to assess the publication bias that may exist in the study. Sensitivity analysis was performed to detect the origin of the heterogeneity.

Information sources: The English databases, "PubMed", "Embase" and the "Cochrane Library" were searched at the beginning of the present research. The team performed the searching by using the items "preoperative chemotherapy", "NACT", "neoadjuvant chemotherapy", plus "response" or "responder" or "responding" or "responsiveness" or "clinical response" or "remission", plus "cervical cancer" or "cervical carcinoma" or "cervical neoplasia". To avoid the data missing, the reference articles in the retrieved articles were also reviewed.

INPLASY registration number: This protocol was registered with the International Platform of Registered Systematic Review and Meta-Analysis Protocols (INPLASY) on 24 February 2022 and was last updated on 24 February 2022 (registration number INPLASY202220107).

\section{INTRODUCTION}

Review question / Objective: Background: Neoadjuvant chemotherapy has been used for treatment of cervical cancer for a long time; however, the role of early nonresponse on prognosis is still confusing. This study was designed to assess its impact on disease-free survival (DFS). Methods: Databases "PubMed", "Embase" 
and the "Cochrane Library" were searched out through May 2020, and both random effects model and fixed effect model were employed to calculate the main pooled results. 12 and Cochrane $Q$ test were used to test the heterogeneity among the studies. Funnel plot with Begg's and Egger's tests was used to assess the publication bias that may exist in the study. Sensitivity analysis was performed to detect the origin of the heterogeneity.

Condition being studied: Cervical cancer has been one of the most common malignant disease in undeveloped areas. Nowadays, clinicians have resorted to neoadjuvant chemotherapy (NACT) plus surgery for the treatment of cervical cancer. And there are several reasons: firstly, NACT can reduce the tumor size, diminish distant metastasis, and facilitate the surgery; secondly, instead of radiotherapy, NACT plus surgery provides the opportunity to have the vaginal function and ovarian function preserved, and patients consequently enjoy better life quality (3). Thirdly, for young women who are eager to have their reproductive function preserved, NACT provides the chance of genital preservation (4-6). Fourth, for women who are pregnant, NACT provides the chance to prolong the duration of pregnancy (7). Thus, NACT has widely been used across the world (8). Recently, quite a number of studies have investigated the prognostic role of NACT's short-term response on long-term survival. However, the results are always disputing (9). Therefore, the present study is designed aiming to give a pooled conclusion by using the published data from the previous studies.

\section{METHODS}

Participant or population: Recently, quite a number of studies have investigated the prognostic role of NACT's short-term response on long-term survival.

Intervention: NACT.

Comparator: responders vs nonresponders.
Study designs to be included: case-control studies, cohort studies.

Eligibility criteria: Three rounds of identification were adopted to collect the necessary studies in the present research. Articles with cervical cancer and therapy were firstly searched out. During the first screening, articles concerning about cervical cancer and chemotherapy were included by reading the titles and abstracts of the articles; otherwise, they would be excluded. During the second screening, articles focusing on cervical cancer and NACT were excluded by reading the result section of the papers as well as the supplementary materials. Meanwhile, the selected articles must fulfill all of our criteria: the articles must be written in English; the articles must be original research articles; all cases in the articles were definitely diagnosed with cervical carcinoma; the included articles must be published in the journals following peerreview disciplines. During the third screening, articles with disease-free survival (DFS) data were included in the final analysis. Newcastle-Ottawa Scale (NOS) was adopted to evaluate the quality of the included studies.

Information sources: The English databases, "PubMed", "Embase" and the "Cochrane Library" were searched at the beginning of the present research. The team performed the searching by using the items "preoperative chemotherapy", "NACT", "neoadjuvant chemotherapy", plus "response" or "responder" or "responding" or "responsiveness" or "clinical response" or "remission", plus "cervical cancer" or "cervical carcinoma" or "cervical neoplasia". To avoid the data missing, the reference articles in the retrieved articles were also reviewed.

Main outcome(s): As described above, the relationship between the $\mathrm{CR}$ and long-term DFS was measured by HR with $95 \%$ confidential interval (Cl).

Quality assessment / Risk of bias analysis: During the process, 12 statistic was adopted in our study to evaluate the 
heterogeneity that may exist in the studies; if 12 got the value larger than $50 \%$, the statistically significant heterogeneity was observed and then the random effect model was used in the study instead of the fixed effect model (23). Meanwhile, Cochrane $Q$ test was also used to assess the heterogeneity; and the fixed effect model should not be adopted when statistical heterogeneity was observed; in this condition, the researchers were obliged to use the random effect model.

Strategy of data synthesis: Forest plot was used to illustrate the pooled result for the $\mathrm{HR}$ and the corresponding $95 \% \mathrm{Cl}$.

Subgroup analysis: According to the previous studies, responder was defined as clinical response (CR) + partial response (PR); at the same time, non-responder was defined as stable disease (SD) + progressive disease (PD). Both RECIST criteria and WHO criteria were adopted to evaluate the CR by previous studies, so in this study we also investigated both criteria. In WHO criteria, tumor response was judged according to bidimensional measurements; in RECIST criteria, tumor response was judged by one dimension measurement. There were slight differences between the two response criteria (14). Both criteria were widely accepted as standard methods in assessing the CR among the field of solid tumor research as well as among cervical cancer.

Sensitivity analysis: Sensitivity analysis was also employed to test the robustness of the pooling result; and also to detect the origin of the heterogeneity.

Country(ies) involved: China.

Keywords: Early response; meta-analysis; neoadjuvant chemotherapy (NACT); recurrence; uterine cervicalneoplasms.

Contributions of each author:

Author 1 - Kecheng Huang.

Author 2 - Xiaoxiong Wu.

Author 3 - Zhilan Chen. 